\title{
Construction of a Genetic Linkage Map of Shiitake Mushroom Lentinula Edodes Strain L-54
}

\author{
Kwan Hoi-Shan and Xu Hai-Lou* \\ Department of Biology, The Chinese University of Hong Kong, Hong Kong SAR, China
}

Received 23 May 2002, Accepted 23 July 2002

\begin{abstract}
From fruiting bodies of $L$. edodes strain L-54, single-spore isolates (SSIs) were collected. Two parental types of L-54 were regenerated via monokaryotization. By means of random-amplified polymorphic DNA (RAPD), DNA samples from L-54, its two parental types, and 32 SSIs were amplified with arbitrary primers. Dedikaryotization was demonstrated, and 91 RAPD-based molecular markers were generated. RAPD markers that were segregated at a 1:1 ratio were used to construct a linkage map of $L$. edodes. This RAPD-linkage map greatly enhanced the mapping of other inheritable and stable markers [such as those that are linked to a phenotype (the mating type), a known gene (priA) and a sequenced DNA fragment (MAT)] with the aid of mating tests, bulked-segregant analysis, and PCR-singlestrand conformational polymorphism. These markers comprised a genetic map of $L$. edodes with 14 linkage groups and a total length of $622.4 \mathrm{cM}$.
\end{abstract}

Keywords: Genetic map, Genetic mapping, Lentinula edodes, Random-amplified polymorphic DNA (RAPD)

\section{Introduction}

Shiitake mushroom, Lentinula edodes, is worldwide the second most popular edible mushroom (Chang, 1999; Chiu et al., 1999). In order to improve the quality and increase the production of $L$. edodes, many studies have been carried out. However, the genetics of $L$. edodes have not been extensively studied. The lack of genetic information has handicapped the breeding of this mushroom.

Only scanty genetic information and a few genetic markers have been available. The traditional methods for generating

*To whom correspondence should be addressed.

Tel: 65-6879-0498; Fax: 65-6772-1976

E-mail: hlxu@sp.edu.sg.

Present address: School of Chemical and Life Sciences, Singapore Polytechnic, 500 Dover Road, Singapore 139651 genetic markers, such as auxotrophic and drug-resistant markers, are time-consuming and require characterization of the mutations before they can be used. There are also not enough earlier molecular markers [e.g., isozyme and DNA restriction fragment length polymorphism (RFLP) basedmarkers of $L$. edodes] to construct a useful genetic map (Royse et al., 1983; Castle et al., 1987). A more contemporary method uses PCR, such as random-amplified polymorphic DNA (RAPD), to generate molecular markers (Williams et al., 1990). In L. edodes, the parental monokaryons and singlespore progeny can be regarded as F1 and F2 generations, respectively (Kwan, 1992). With this "well-planned cross", RAPD polymorphisms among a reasonable number of singlespore isolates (SSIs) can be produced, analyzed, and linked in order to construct a genetic map.

The objectives of this study are as follows: (1) Construct a genetic map of L. edodes, mainly by the RAPD method with standard mapping. (2) Locate phenotypic markers with the aid of bulked-segregant analysis, a known gene and sequenced DNA fragment by means of PCR-single-strand conformational polymorphism (PCR-SSCP).

\section{Materials and Methods}

Strain and growth conditions Professor Chang Shu-Ting (Department of Biology, the Chinese University of Hong Kong) kindly provided the L. edodes dikaryotic strain L-54. Working stocks were grown on PDA plates $(24.0 \mathrm{~g} / \mathrm{l}$ of potato dextrose broth and $15.0 \mathrm{~g} / \mathrm{l}$ of Difco Bacto agar) at $26^{\circ} \mathrm{C}$ for 7 (of dikaryon), or 14 16 (of monokaryon) days. For DNA extraction, mycelia were inoculated in a PDB medium (24.0 g/l of potato dextrose broth) at $26^{\circ} \mathrm{C}$ for 10 days (of dikaryon), or 15 20 (of monokaryon) days with shaking at $250 \mathrm{rpm}$.

Single-spore isolation Basidiospores were isolated from freshfruiting bodies by holding the cap, gills downward, in a sterile petri dish for $8 \mathrm{~h}$ at room temperature. Spore deposits were suspended in distilled water and spread on PDA plates. After incubation for 3-4 days at $26^{\circ} \mathrm{C}$ in the dark, individually-germinated basidiospores were transferred onto fresh PDA plates. SSIs were distinguishable 
from dikaryons by their slow growth and the absence of clamp connections.

Recovery of parental types Two parental types were recovered from L-54 dikaryon by protoplast isolation and regeneration (Zhao and Chang 1993). Two parental monokaryons (P1 and P2) were identified by their positive-mating reaction when they mated with each other.

DNA extraction DNA samples were extracted from the mycelia of strains L-54, P1 and P2, and SSIs by the method of Lee and Taylor (1990), which was followed by RNase treatment and cesium chloride precipitation (Yoon et al. 1991). The concentration and purity of the DNA preparations were assayed by spectrophotometric measurements (Milton Roy Spectronic 3000, GeneQuant RNA/DNA Calculator) and $0.7 \%$ agarose gel electrophoresis in a $1 \times$ TBE buffer (prepared from a 10× TBE stock solution containing $0.9 \mathrm{M}$ Tris base, $0.9 \mathrm{M}$ boric acid, and $2 \mathrm{mM}$ EDTA).

Generation of RAPD markers RAPD markers were generated by RAPD amplification of DNAs of strains P1 and P2, and then 32 SSIs. RAPD protocol was an optimization of that described by Sobral and Honeycutt (1993) with the AmpliTaq Stoffel fragment (Perkin-Elmer Cetus) as the DNA polymerase. All of the tests were in a $50 \mathrm{ml}$ reaction that contained $1 \times$ Stoffel buffer $(10 \mathrm{mM}$ Tris$\mathrm{HCl}, 10 \mathrm{mM} \mathrm{KCl}, \mathrm{pH} 8.3), 6.0 \mathrm{mM} \mathrm{MgCl} 2,0.2 \mathrm{mM}$ dNTP, $50 \mathrm{ng}$ template DNA, $0.4 \mathrm{mM}$ primer, and $5 \mathrm{U}$ Stoffel fragment. The reaction mixture was subjected to 40 cycles in a PTC-100 Programmable Thermal Controller (MJ Research) through a thermal profile of $94^{\circ} \mathrm{C}$ for $1 \mathrm{~min}, 35^{\circ} \mathrm{C}$ for $1 \mathrm{~min}$, and $72^{\circ} \mathrm{C}$ for $2 \mathrm{~min}$, followed by a 20-min elongation. RAPD products were detected by electrophoresis on $3 \%$ Nuseive:Seakem $(3: 1)$ agarose gel and stained with $0.5 \mathrm{mg} / \mathrm{ml}$ ethidium bromide for $15 \mathrm{~min}$, then destained with distilled water for $10 \mathrm{~min}$. The gel was transferred to a UV transilluminator and photographed with Polaroid 667 film (ASA3000). The primers (Operon 10-mer Kit A-D, OPA-OPD, Operon Technologies) were 10-base oligonucleotides with arbitrary sequences without self-complementary ends. Their $\mathrm{G}+\mathrm{C}$ content was $60-70 \%$.

Linkage analysis A series of pairwise chi-square $\left(\chi^{2}\right)$ tests of independent assortment were conducted to screen for RAPD markers with the expected $1: 1$ segregation pattern among the progeny (Weir 1990). The RAPD markers that showed significant deviation $(P<0.05)$ from the null hypothesis of the independent assortment were not used for the subsequent analysis.

The linkage of the markers was tested by LOD Score (the threshold of Lod score was 3.00). The MAPMAKER/MAP version $3.0 \mathrm{~b}$ computer program (Whitehead Institute for Biomedical Research) performed the linkage analysis. This program uses an efficient algorithm that allows a simultaneous multipoint analysis of any number of loci (Lander et al., 1987). MAPMAKER does not recognize linkages for markers in repulsion, so the repulsion-phase linkages can be detected by analyzing re-coded data (i.e., presence re-coded to absence, and vice versa) together with the original data set using the F2 backcross data type (http://www-genome.wi.mit.edu/ genome_software/other/mapmaker.html). Grouping of the marker loci was conducted using the "GROUP" (two-point analysis) and "COMPARE" commands. The "ORDER" command was used to determine a linear order using a multi-point analysis.

Mating tests Mating types of the two parental monokaryons, P1 and $\mathrm{P} 2$, were designated as $\mathrm{A} 1 \mathrm{~B} 1$ and $\mathrm{A} 2 \mathrm{~B} 2$, respectively. The mating type of an SSI was determined by mating reactions between this SSI and two parents. It was confirmed by mating reactions between all of the SSIs. To test for a mycelial interaction, two 5$\mathrm{mm}^{2}$ plugs of agar and mycelia from two monokaryons were placed 1.5-2.0 $\mathrm{cm}$ apart on a 9-cm petri dish that contained a PDA medium, and cultivated at $26^{\circ} \mathrm{C}$ for 7-15 days. Mycelial interactions were observed continuously and recorded as positive, common A (Flat), common B (Barrage), and negative (Miles \& Chang 1986). Morphological characteristics of 'common A' and 'common B' were compared by reference mating.

Bulked-segregant analysis Bulked-segregant analysis or pooled sampling is a method for rapid identification of markers that are linked to any specific gene or genomic region (Michelmore et al., 1991). The success of the bulked-segregant analysis depends on the genetic divergence between the two parents in the target region. Within each pool, individuals are identical for the trait, but are arbitrary for all others. In this study, the bulked-segregant analysis was carried out to isolate RAPD markers that were closely linked to the mating type factors A and B of $L$. edodes. Sixteen SSIs were grouped into 4 pools. Each pool had 4 SSIs with the same mating type. DNAs from 4 pooled samples were extracted and amplified by RAPD. The polymorphisms were confirmed to link to the mating type loci by RAPD amplification of the 32 SSIs segregation with the same primer pairs. Although there were only 4 SSIs per pool to a specific mating type factor (either A or B), 8 individuals were used for analysis because the mating system of $L$. edodes is bifactorial. Theoretically, for a dominant marker that segregates in the F2 population, the probability of a pool of 8 individuals that have a band, and a second pool of equal size does not have this band, is $2 \times(1 / 4)^{8} \times\left[1-(1 / 4)^{8}\right]=3.0 \times 10^{-5}$, when the locus is unlinked to the target gene.

PCR-single-strand conformational polymorphism (PCR-SSCP) PCR-SSCP is a genetic-screening method to rapidly detect single nucleotide substitutions in genomic DNA or cDNA that are amplified by PCR (Orita et al., 1989). The double-stranded DNA target sequence is denatured to 2 single-stranded DNA molecules, which are separated by polyacrylamide gel electrophoresis (PAGE) under non-denaturing conditions. Fragments of equal length that differ in sequence migrate to different positions, according to their conformational changes. The separated bands of the single-stranded DNA indicate the presence of mutations.

In order to map a known gene (priA) (Kajiwara et al., 1992) and a sequenced DNA fragment (MAT) (Leung et al., 1995), the fragments of interest that were inherited in 32 SSIs were amplified by PCR with designed primers (Table 1), then subjected to a PCRSSCP analysis. One $\mathrm{ml}$ that contained approximately $100 \mathrm{ng}$ of the PCR product was mixed with $1 \mathrm{ml}$ of the denaturing Solution $(98 \%$ formamide containing $0.05 \%$ bromophenol blue and $0.05 \%$ xylene cyanol). It was incubated at $95^{\circ} \mathrm{C}$ for $3 \mathrm{~min}$, then quickly placed in an ice/water bath to cool for a few minutes. Before starting the 
Table 1. Lengths of the target sequences and primer pairs used by PCR-SSCP.

\begin{tabular}{cccc}
\hline Target DNA & Target Length $(\mathrm{bp})$ & Primer & Sequence \\
\hline \multirow{2}{*}{ priA } & 302 & $\begin{array}{l}\text { Upper } \\
\text { Lower }\end{array}$ & $\begin{array}{l}\text { CTACCCCCAACAAAGGAAATG } \\
\text { ACACTACGAAACAAGAATCAG }\end{array}$ \\
\hline \multirow{2}{*}{ MAT } & 200 & $\begin{array}{l}\text { Upper } \\
\text { Lower }\end{array}$ & $\begin{array}{l}\text { ATGCTCACCAACAATCAACC } \\
\text { CTGGTGTTGCGGGAGTGAGG }\end{array}$ \\
\hline
\end{tabular}

electrophoresis, PhastGel media [including PhastGel Homogeneous 12.5 (12.5\% polyacrylamide) and PhastGel Native Buffer Strips (0.88 M L-alanine, $0.25 \mathrm{M}$ Tris- $\mathrm{HCl}, \mathrm{pH} 8.8,3 \%$ agarose)] were placed in the PhastSystem (Pharmacia Biotech), and the stand-by temperature $\left(4^{\circ} \mathrm{C}\right)$ was set. A $100 \mathrm{Vh}$ of pre-run was performed to generate a continuous-buffer system. Soon after denaturation, $0.8 \mu \mathrm{l}$ of the sample was pipetted onto an upturned $8 / 1 \mu \mathrm{l}$ sample applicator and placed in the PhastSystem. The separation began to run immediately. According to the PCR fragment size, the separation was run from $150 \mathrm{Vh}$ (for $200 \mathrm{bp}$ ) to $250 \mathrm{Vh}$ (for 300 bp). After the separation-running, the gel was fixed and stained.

\section{Results and Discussion}

Generation of RAPD markers Primer pairs, rather than single primers, were used in this study. With 86 primer pairs, 7-10 amplified fragments were generated per primer pair. The first step of RAPD marker screening was the primer pair selection. A total of 48 primer pairs were tested; 27 of them were selected, based on their successful generation of polymorphic RAPD bands from two parents. This step was also the dedikaryotization demonstration: with the same primer pairs, the RAPD profiles of $\mathrm{P} 1$ and $\mathrm{P} 2$ could be distinguished as containing a subset of the bands that were seen in L-54.

With the 27 primer pairs, RAPD markers were identified by detection of the polymorphic fragments that were amplified among 32 SSIs. Each primer pair generated 3-4 polymorphic bands on average. The markers ranged in size from 70 to 740 bp. Figure 1 shows a typical RAPD amplification among 32 SSIs with the primer pair of OPC-07 and 08. There were two polymorphic bands of $182 \mathrm{bp}$ and $340 \mathrm{bp}$ in size. Each marker was given a name that was designated by a combination of the primer names and the size (in bp) of the polymorphic band. For clarity, the serial alphabet "a-t" were used in place of the primer numbers "01-20" in the Operon 10-mer Kits. For example, a 182 bp band that was produced with the primer pair of OPC-07 and 08 was designated as $\mathrm{CgCh} 182$.

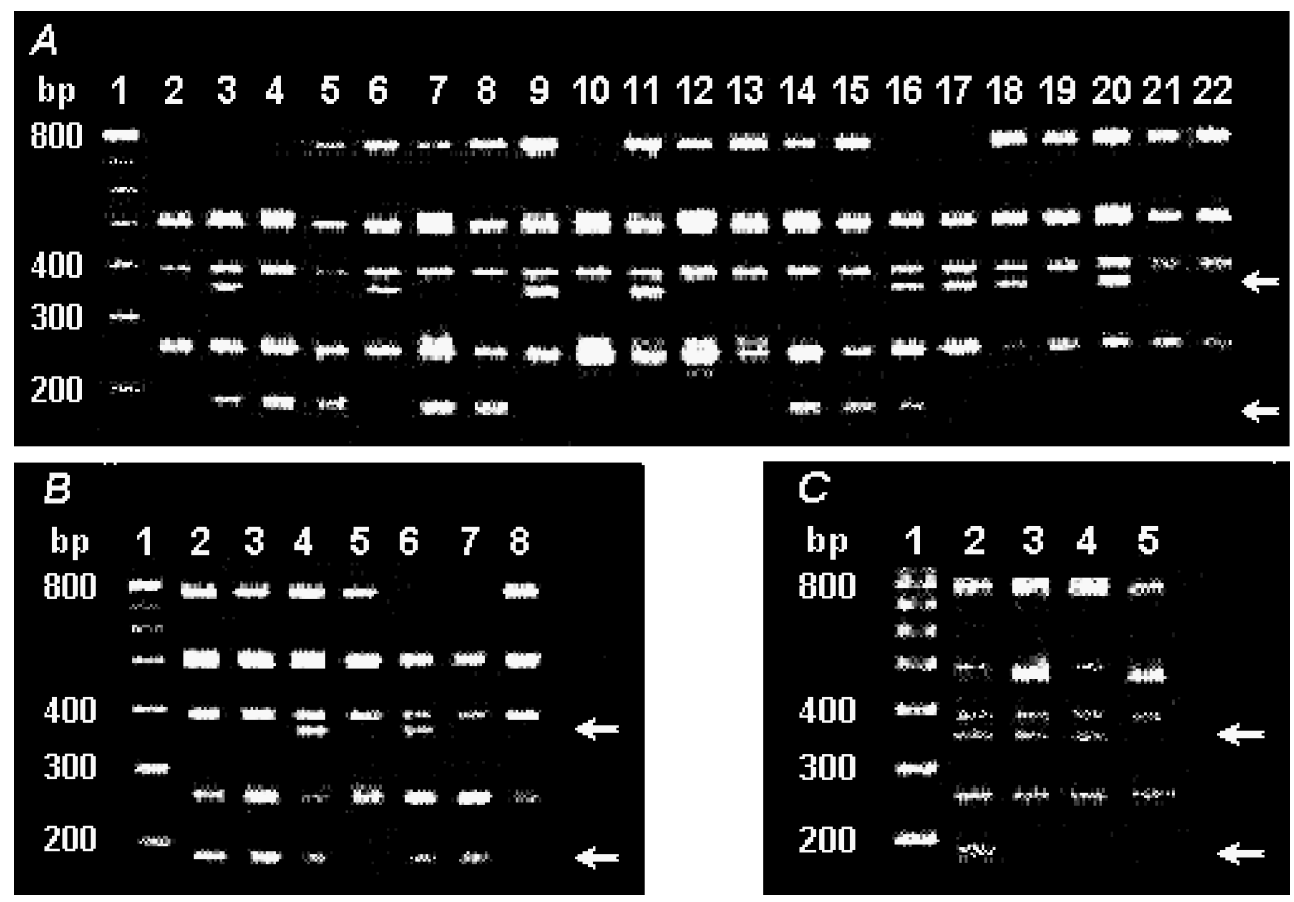

Fig. 1. RAPD bands of 32 SSIs with Operon 10-mer primer of OPC-07 and OPC-08 as the primer pair. Each lane 1 is the size marker (100-bp ladder); lane 2 22 in $A$, lane $2 \sim 8$ in $B$, and lane 2 5 in $C$ are SSIs. Arrows indicate the RAPD markers, CgCh182 and $\mathrm{CgCh} 340$. 


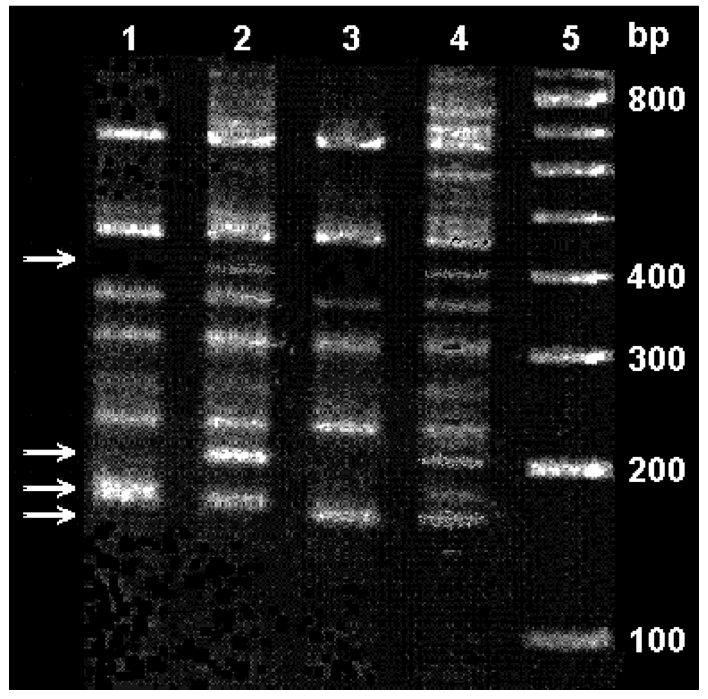

Fig. 2. RAPD bands of pooled samples of SSIs with the primer pair of OPB-05 and OPC-05. Land 1 4 are pools with mating types of $\mathrm{A} 1 \mathrm{~B} 1, \mathrm{~A} 1 \mathrm{~B} 2, \mathrm{~A} 2 \mathrm{~B} 1$ and $\mathrm{A} 2 \mathrm{~B} 2$, respectively; lane 5 is the size marker (100-bp ladder). Arrows indicate the bulked segregant analysis-based RAPD markers, BeCe160, BeCe170, $\mathrm{BeCe} 210$, and $\mathrm{BeCe} 400$.

Linkage analysis and linkage map construction RAPD with primers of short oligonucleotides enhanced the development of the genetic linkage map. A total of 91 polymorphic bands were identified; 78 had the $1: 1$ segregation ratio at the 0.05 level of significance. Of the 78 markers that were analyzed, 62 were mapped to 14 linkage groups by MAPMAKER/MAP. Two markers were considered linked when the Lod score between them was larger than 3.00. Relative positions of these markers were estimated by maximum likelihood. The remaining 16 RAPD markers were difficult to score with confidence, and were not included on the map. In most cases, the separate RAPD markers that were amplified with the same primer pair assorted independently. Sometimes, however, these fragments demonstrated linkage or even tight linkage.

Once a genetic map is constructed, some traits can be extensively characterized and inheritance patterns can be determined. In this study, the following strategies were employed to map the mating type factors, priA gene, MAT fragment, and the growth rate loci of $L$. edodes. The RAPD linkage map facilitated the mapping of these genetic loci.

Mapping of mating type loci Mating type genes are essential to the cultivation of $L$. edodes, because the breeding of this mushroom is always limited by the incompatibility of its mating system (Chang \& Miles, 1991). L. edodes has a bifactorial-incompatibility mating system that is mediated by two phenotypic, loci A and B. This situation is similar to that of Schizophyllum commune in that it has a multiallelictetrapolar mating system, where the allelic forms of the incompatibility locus A are passed on to sexual progenies independently from those of the incompatibility locus B (Chang \& Miles, 1991).

The tetrapolar incompatibility of $L$. edodes was confirmed in mating tests, because only the mating system with two incompatibility factors could give the four different mating patterns -A1B1, A2B2, A1B2 and A2B1- that are found within SSIs of L-54. From the mating types of 32 SSIs that were used in the RAPD amplification, the mating types loci A and $\mathrm{B}$ were analyzed by the MAPMAKER/MAP program with the 62 RAPD markers on the linkage map, and localized on linkage groups III and II, respectively (see Fig. 4).

Mapping of markers linked to mating-type genes RAPD amplification of the 4 pooled DNA samples generated 11 bulked-segregant analysis-based RAPD markers. Of the 11 markers that were analyzed by RAPD amplification of 32 SSIs, 3 were rejected by the segregation data analysis. Eight markers were confirmed to link to mating-type loci by a MAPMAKER/MAP linkage analysis; 3 of them were new markers that were not obtained by the RAPD analysis of P1 and P2. Four SSIs per pool was a practical and efficient pool size. Figure 2 is an example of the RAPD amplification of the pooled DNAs with the primer pairs OPB-05 and OPC-05, where 4 polymorphic bands were produced. BeCe160 and BeCe400 were previously observed in the first round of the RAPD amplification. BeCe170 was a new marker that was confirmed to link to the mating-type locus A, but $\mathrm{BeCe} 210$ was rejected.

A bulked-segregant analysis consolidated the linkage groups II and III by identifying the markers at the positions around the mating type loci. About $90 \%$ of the 11 linkedbulked-segregant analysis-based markers had a distance of less than $10.0 \mathrm{cM}$ away from the mating types locus A or B, and $40 \%$ were closely linked (map distance $<5.0 \mathrm{cM}$ ).

Mapping of the priA gene and MAT fragment Since the mapping of known genes or sequenced DNA fragments facilitates the identification of candidate genes or DNA fragments for mutations, a strategy for mapping genes or DNA fragments was developed using PCR-SSCP. With DNAs from strains L-54, P1 and P2, and 32 SSIs as the templates, and designed upper and lower primers (from published sequences) of priA and MAT as the primer pairs, the target sequences were amplified by PCR, and analyzed by SSCP. Figure 3 shows the MAT polymorphism by a SSCP analysis with SSIs of L-54 as the inherited progeny.

The first cloned gene of L. edodes, priA, may play a role at the beginning of fruiting (Kajiwara et al., 1992). Primordia contain higher levels of the priA transcript than preprimordial mycelia and mature-fruiting bodies. A 302-bp fragment of the priA gene was amplified by PCR and analyzed by SSCP with 32 SSIs as the inherited progeny. The segregation data from SSCP were tested by chi-square calculation $\left(\chi^{2}=0.5\right)$. Through the likelihood estimation by MAPMAKER/MAP, 

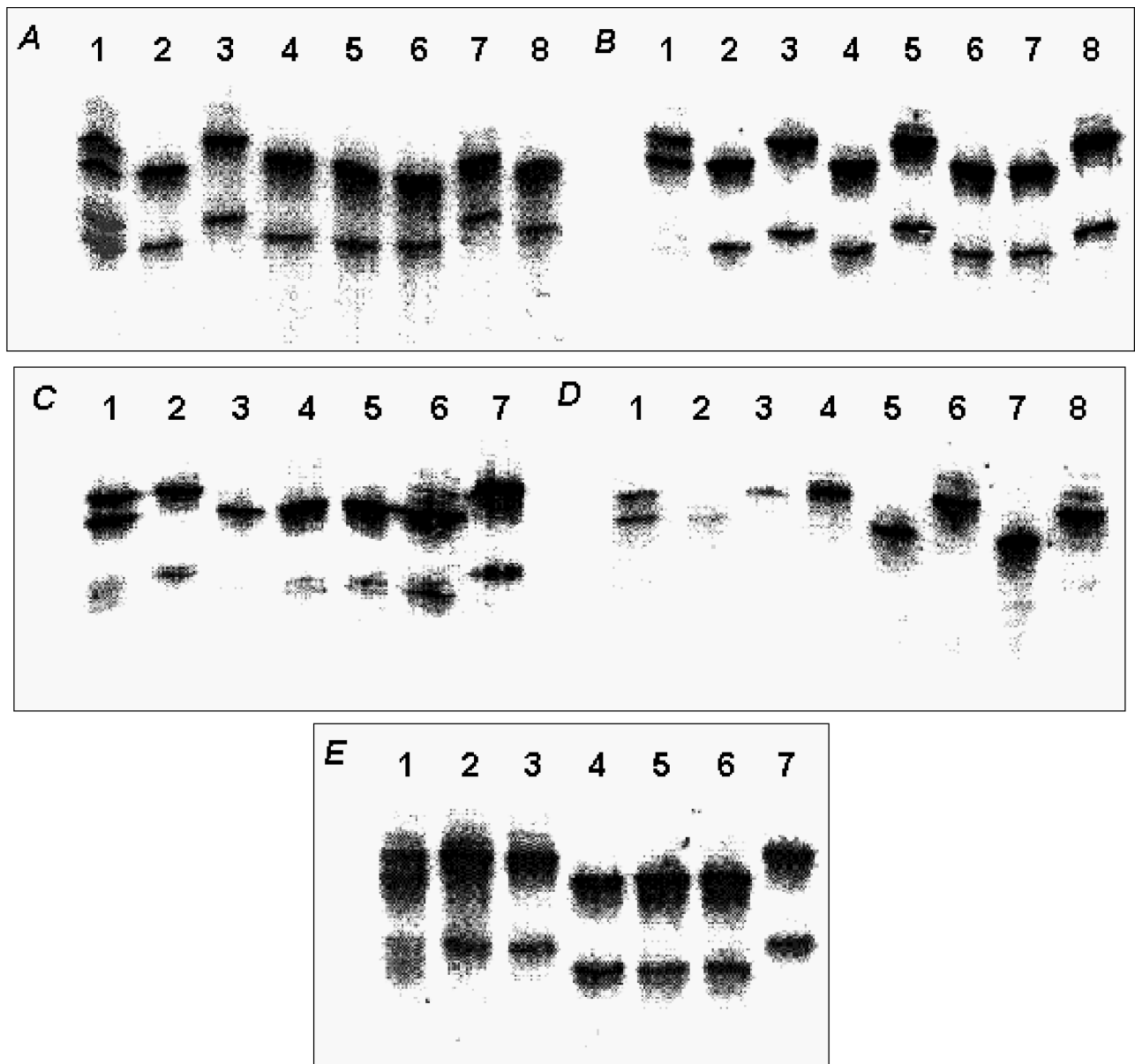

Fig. 3. Detection of MAT polymorphism by PCR-SSCP analysis in SSIs of L-54. Each lane 1 is L-54; lane 2 \& 3 in $A$ are P1 \& P2, respectively; the rest are SSIs.

priA locus was localized on the linkage group III of the linkage map of $L$. edodes at the position of $23.1 \mathrm{cM}$ from AiCi500 (see Fig. 4).

The MAT fragment is a differentially-expressed cDNA that is amplified by RNA arbitrarily-primed PCR (RAP) from the RNAs of vegetative mycelia, primordia, as well as young and mature fruiting bodies of the strain L-54 (Leung et al., 2000). A 200-bp of the MAT fragment was picked for a PCR-SSCP analysis. MAT locus was mapped on the linkage group IX at the position of $12.2 \mathrm{cM}$ from AtBt210 (see Fig. 4).

Genetic map of $\boldsymbol{L}$. edodes strain L-54 The genetic map of the L. edodes strain L-54 with 14 linkage groups is summarized in Fig. 4. The map included 62 RAPD markers, 3 bulked-segregant analysis-based RAPD markers, 2 phenotypic loci (mating type factors $\mathrm{A}$ and $\mathrm{B}$ ), 1 known gene locus (priA), and 1 sequenced DNA fragment locus (MAT). Linkage groups ranged in length from 0.1 to $122.6 \mathrm{cM}$ with a total length for the map of $622.4 \mathrm{cM}$. The distance between the adjacent markers ranged from 0.0 to $30.3 \mathrm{cM}$ with an average of $9.0 \mathrm{cM}$.

It was reported that with a contour-clamped homogeneous electric field (CHEF) gel electrophoresis, the chromosomesized DNA was separated into 8 bands in L. edodes (Arima \& Morinaga, 1993). The genetic map of L. edodes in this study contained 14 linkage groups, which was 6 more than the number of haploid chromosomes. The deviation was probably due to some linkage groups that could be linked together when more SSIs were analyzed and more genetic markers were generated.

Based on the consideration of the unfilled gaps, the total length of the L. edodes genome (in $\mathrm{cM}$ ) can be estimated (Postlethwait et al. 1994). The unfilled gap distance from the two end markers on each chromosome to their adjacent telomeres is estimated to be $\mathbf{2}$ telomeres per chromosome $\times$ $\boldsymbol{d} / \mathbf{2} \times \boldsymbol{C}$, where $\boldsymbol{C}$ is the number of chromosomes, $\boldsymbol{d}$ is the average interval, and $d / 2$ indicates an average distance from the end marker to the telomere. The distance for other unfilled gaps is estimated to be $(\boldsymbol{L}-\boldsymbol{C}+\boldsymbol{N}) \times \boldsymbol{d}_{\mathrm{m}}$, where $\boldsymbol{N}$ is the number of unlinked markers, $\boldsymbol{L}$ is the number of linkage groups, and $\boldsymbol{d}_{\mathrm{m}}$ is the maximum interval that is accepted in the map. Therefore, the estimate of the complete map size of $L$. edodes is about $622.4+(8 \times 2 \times 9.0 / 2)+(14-8+16) \times 30.3$, or $1,360 \mathrm{cM}$. 

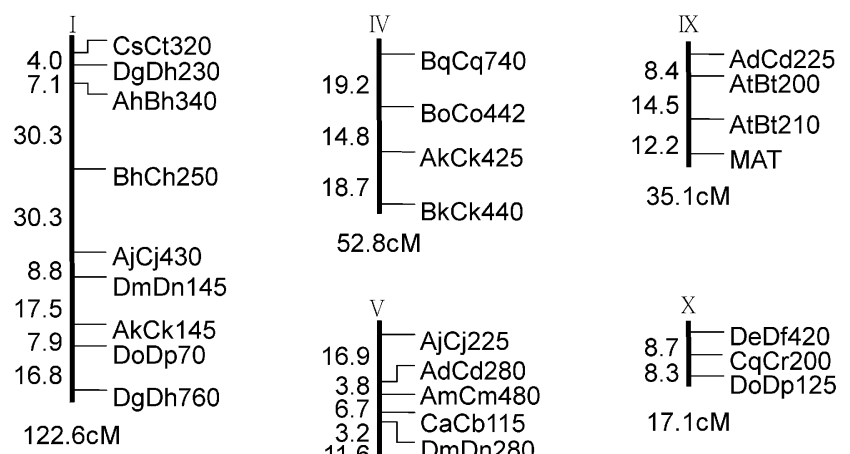

$52.8 \mathrm{cM}$
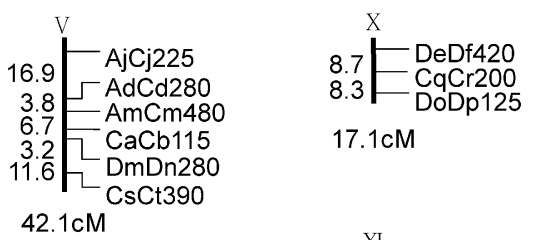

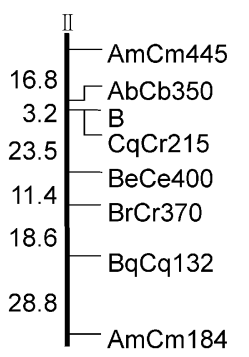

$102.4 \mathrm{cM}$

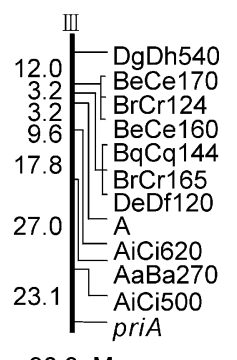

$96.3 \mathrm{cM}$

Fig. 4. Genetic map of Lentinula edodes strain L-54 with 14 linkage groups, including 62 RAPD markers, 3 bulked segregant analysis-based RAPD markers, 2 phenotypic loci (mating type factors A and B), 1 known gene locus (priA) and 1 sequenced DNA fragment locus (MAT). Roman numerals at the top are numbers of linkage groups. Names indicated on the right side are markers of each locus. Distances indicated on the left side are estimated percent recombination between the markers (in $\mathrm{cM}$ ), which are depicted as the maximum-likelihood solutions provided by MAPMAKER/MAP (version 3.0b).

Another approach to estimate the size of a complete map is based on a partial-linkage map and consideration of the marker density (Hulbert et al., 1988). Supposing the complete map length is $\boldsymbol{G}$ (in cM), let $\boldsymbol{P}(\boldsymbol{T}, \boldsymbol{G})$ denote the probability that the Lod score for the random pair of markers $(\boldsymbol{Z})$ will not be less than the Lod score threshold $(\geq T)$, and let $N$ denote the number of markers that are genotyped. Then the expected number of marker pairs that have $Z \geq T$ is $C_{\mathrm{N}}{ }^{2} P(T, G)=[(\mathbf{1} / \mathbf{2})$ $N(N-1)] P(T, G)$. If the actual number of such pairs is $K, P(T$, $G)=K /[(\mathbf{1} / \mathbf{2}) \boldsymbol{N}(\boldsymbol{N}-\mathbf{1})]$, then $P(T, G)$ can be given by the chance that a randomly-chosen pair of loci lies within $X \mathrm{cM}$, $\boldsymbol{P}(\boldsymbol{T}, \boldsymbol{G})=\mathbf{2} \boldsymbol{X} / \boldsymbol{G}$, where $\boldsymbol{X}=\boldsymbol{X}(\boldsymbol{T})$ is the distance between the two markers for which the expected Lod score is $\boldsymbol{T}$. Accordingly, the estimate of the genome size is given by $\boldsymbol{G}=$
$N(N-1) X / K$. In this study, if $X$ is $\leq 18 \mathrm{cM}(T=3.00)$, then the estimate $\boldsymbol{G}$ is $1,200 \mathrm{cM}$. If $\boldsymbol{X}$ is $\leq 24 \mathrm{cM}(\boldsymbol{T}=2.00)$, then $\boldsymbol{G}$ is estimated to be $1,000 \mathrm{cM}$. These estimates are consistent with that based on the consideration of the unfilled gaps. The length of the complete map on average is given by $1,200 \mathrm{cM}$, which suggests that the current genetic map covers more than $50 \%$ of the whole genome of L. edodes.

The utility of a genetic map is related to its degree of completeness. Assuming that the $N$ markers are randomly distributed in a genome the size of $\boldsymbol{G} \mathrm{cM}$, if there is at least one marker that lies within $\boldsymbol{d} \mathrm{cM}$ of the genome, then each marker will sweep an area of $\mathbf{2} \boldsymbol{d} \mathrm{cM}$; therefore, the $\boldsymbol{N}$ markers

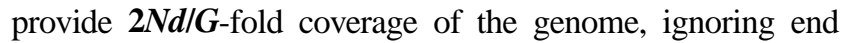
effects (Jacob et al., 1991). So, the proportion of the genome within $\boldsymbol{d} \mathrm{cM}$ of at least one marker is approximately $\mathbf{1}-\exp (-$ $2 N d / G)$. As the genetic length of $L$. edodes is estimated to be $1,280 \mathrm{cM}$, it is expected that about $86 \%$ of the genome should be located within $20 \mathrm{cM}$ of a marker, about $77 \%$ should be located within $15 \mathrm{cM}$ of a marker, and $62 \%$ should be within $10 \mathrm{cM}$ of a marker. The average distance between the adjacent markers is $9.0 \mathrm{cM}$, the fact that 29 of the 91 RAPD markers remain unlinked to any other marker supports this expectation.

The average map distance of one $\mathrm{cM}$ in $\mathrm{kb}$ of DNA is usually estimated by comparing the size of a physical map (in $\mathrm{kb}$ ) to the length of its genetic map (in cM). Because the karyotype analysis showed that the total genome size of $L$. edodes was about $33 \mathrm{Mb}$ (Arima \& Morinaga, 1993), then this estimate of the genetic map length gives an average of $27 \mathrm{~kb}$ per $\mathrm{cM}$. As references, in fungi, one $\mathrm{cM}$ represents $25 \mathrm{~kb}$ in Bremia lactucae (Hulbert et al., 1988), $23 \mathrm{~kb}$ in Cochliobolus heterostrophus (Tzeng et al., 1992), and $40 \mathrm{~kb}$ in Magnaporthe grisea (Skinner et al., 1993).

\section{References}

Arima, T. and Morinaga, T. (1993) Electrophoretic karyotype of Lentinus edodes. Transac. Mycol. Society Japan 34, 481-485.

Castle, A. J., Horgen, P. A. and Anderson, J. B. (1987) Restriction fragment length polymorphisms in the mushrooms Agaricus brunnescens and Agaricus bitorquuis. Appl. Environ. Microbiol. 53, 816-822.

Chang, S. T. (1999) World production of cultivated edible and medicinal mushrooms in 1997 with emphasis on Lentinula edodes in China. Int. J. Med. Mushroom 1, 291-300.

Chang, S. T. and Miles, P. G. (1991) Edible mushrooms and their cultivation. CRC Press, Boca Raton, FL.

Chiu, S. W., Wand Z. M., Chiu, W. T., Lin, F. C. and Moore, D. (1999) An integrated study of individualism in Lentinula edodes in nature and its implication for cultivation strategy. Mycol. Res. 103, 651-660.

Hulbert, S. H., Hott, T. W., Legg, E. J., Lincoln, S. E., Lander, E. S. and Michelmore, R. W. (1988) Genetic analysis of the fungus, Bremia lactucae, using Restriction Fragment Length Polymorphisms. Genetics 120, 947-958.

Jacob, H. J., Lindpaintner, K., Lincoln, S. E., Kusumi, K., Bunker, R. K., Mao, Y. P., Ganten, D., Dzau, V. J. and Lander, E. S. (1991) Genetic mapping of a gene causing hypertension in the 
stroke-prone spontaneously hypertensive rat. Cell 67, 213-224.

Kajiwara, S., Yamaoka, K., Hori, K., Miyazawa, H., Saito, T., Kanno, T. and Shishido, K. (1992) Isolation and sequence of a developmentally-regulated putative novel gene, priA, from the basidiomycete Lentinus edodes. Gene 114, 173-178.

Kwan, H. S. (1992) Application of arbitrarily-primed polymerase chain reaction in molecular studies of mushroom; in: Proceedings of the International Symposium on Recent Topics in Genetics, Physiology and Technology of the Basidiomycetes, Miyaji, M., Suzuki, A. and Nishimura, K. (eds.), pp. 87-92. Chiba, Japan.

Lander, E. S., Green, P., Abrahamson, J., Barlow, A., Daly, M. J., Lincoln, S. L. and Newburg, L. (1987) MAPMAKER: an interactive package for constructing primary genetic linkage maps of experimental and natural populations. Genomics 1, $174-181$

Lee, S. B. and Taylor, J. W. (1990) Isolation of DNA from fungal mycelia and single spores; in PCR Protocol, Innis, M. A., Gelfand, D. V., Sninsky, J. J. and White, T. J. (eds.), pp. 282287. Academic Press, New York.

Leung, G. S. W., Zhang, M., Xie, W. J. and Kwan, H. S. (2000) Differentially-expressed genes during the development of the basidiomycete Lentinula edodes identified by RNA fingerprinting. Mol. Gen. Genet. 262, 977-990.

Michelmore, R. W., Paran, I. and Kesseli, R. V. (1991) Identification of markers linked to disease-resistance genes by Bulked Segregant Analysis: A rapid method to detect markers in specific genomic regions by using segregation populations. Proc. Natl. Acad. Sci. USA 88, 9828-9832.

Miles, P. G. and Chang, S. T. (1986) The collection and conservation of genes of Lentinus; in Cultivating Edible Fungi, West, P. J., Royse, D. J. and Beelman, R. B. (eds.), pp. 227234. Elsevier, Amsterdam.

Orita, M., Iwahana, H., Kanazawa, H., Hayashi, K. and Sekiya, T. (1989) Detection of polymorphisms of human DNA by gel electrophoresis as single strand conformation polymorphisms. Proc. Natl. Acad. Sci. USA 86, 2766-2770.

Postlethwait, J. H., Johnson, S. L., Midson, C. N., Talbot, W. S., Gates, M., Ballinger, E. W., Africa, D., Andrews, R., Carl, T., Eisen, J. S., Horne, S., Kimmel, C. B., Hutchinson, M., Johnson, M. and Rodriguez, A. (1994) A genetic linkage map for the zebrafish. Science 264, 699-703.

Royse, D. J., Spear, M. C. and May, B. (1983) Single and joint segregation of marker loci in the shiitake mushroom, Lentinus edodes. J. General Appl. Microbiol. 29, 217-222.

Skinner, D. Z., Budde, A. D., Farman, M. L., Smith, J. R., Leung, H. and Leong, S. A. (1993) Genome organization of Magnaporthe grisea: genetic map, electrophoretic karyotype, and occurrence of repeated DNAs. Theor. Appl. Genetics 87, 545-557.

Sobral, B. W. S. and Honeycutt, R. J. (1993) High output genetic mapping of polyploids using PCR-generated markers. Theor. Appl. Genetics 86, 105-112.

Tzeng, T. H., Lyngholm, L. K., Ford, C. F. and Bronson, C. R. (1992) A restriction fragment length polymorphism map and electrophoretic karyotype of the fungal maize pathogen Cochliobolus heterostrophus. Genetics 130, 81-96.

Weir, B. S. (1990) Genetic Data Analysis: Methods for Discrete Population Genetic Data. Sinauer Associates, Sunderland, MA.

Williams, J. G. K., Kubelik, A. R., Livak, K. J., Rafalski, J. A. and Tingey, S. V. (1990) DNA polymorphisms amplified by arbitrary primers are useful as genetic markers. Nucl. Acids Res. 18, 6531-6535.

Yoon, C. S., Glawe, D. A. and Shaw, P. D. (1991) A method for rapid small-scale preparation of fungal DNA. Mycologia 6, 835-838.

Zhao, J. and Chang, S. T. (1993) Monokaryotization by protoplasting heterothallic species of edible mushrooms. World J. Microbiol. Biotech. 9, 538-543. 